\title{
Muon Scattering Tomography using Drift Chamber Detectors
}

\author{
Jonathan BURNS*, Steve Quillin, Matthew Stapleton, Chris Steer \\ AWE plc, Aldermaston, Reading, United Kingdom, RG7 4PR \\ E-mail: jon.burns@awe.co.uk
}

\begin{abstract}
Muon scattering tomography (MST) allows the identification of shielded high atomic number, or $\mathrm{Z}$ materials by measuring the scattering angle of atmospheric cosmic ray muons passing through an inspection region. Materials of different densities can be differentiated using this technique as muons interacting with high- $Z$ materials are scattered to a greater degree by multiple Coulomb scattering than low- $\mathrm{Z}$ materials. This scattering is determined experimentally by detecting the incoming and outgoing trajectory of the muon as it enters and exits the inspection volume. This technique is particularly useful for imaging volumes that are difficult to inspect visually. AWE is primarily interested in investigating the use of MST for scanning cargo containers, as the technique could be used for the detection the presence of radiological material without introducing an artificial radiation source.

AWE has developed a MST experimental system based on drift chamber technology. This experiment was constructed of two detector modules, each comprising four layers of four adjacent drift chambers, positioned above and below an inspection volume. The technical challenges of producing the experimental system with a resolution capable of accurately determining the muon scattering angle within a few milliradians will be detailed and the experimental results obtained discussed.
\end{abstract}

Technology and Instrumentation in Particle Physics 2014

2-6 June, 2014

Amsterdam, the Netherlands

\footnotetext{
* Speaker.
} 


\section{Introduction}

Muons are produced when cosmic rays interact with particles in the Earth's atmosphere, creating a number of short-lived pions, which readily decay into muons, a charged particle with a mass 200 times that of an electron. Due to the relatively long half life and velocity of muons they are capable of reaching the Earth's surface. Muon's reach sea level with a flux of 1 per $\mathrm{cm}^{2}$ per minute, with an average energy of $3 \mathrm{GeV} / \mathrm{c}$. Muons interact with matter via multiple Coulomb scattering, with an angular distribution that is approximately Gaussian [1]. The magnitude of this scattering is used to infer the density of the materials along the muon path.

A system designed to screen cargo using MST techniques operates by accurately measuring the muon scattering from objects within a particular volume [2][3]. In practice particle tracking detectors are positioned above and below an inspection volume to determine the trajectory of the muon as it enters and exits this region. The point of closest approach (PoCA) of these incoming and outgoing trajectories is used to localise the point at which most of the muon scattering within the volume occurred. A three dimensional image of an inspection volume is created by dividing the inspection volume into a voxelised grid. Each of the PoCAs is localised to particular voxel, with the total number of PoCAs in a particular voxel being recorded. At the end of the scan period, parts of the volume containing high density materials will be identified as being in parts of the grid with the greatest number of recorded PoCAs. A number of techniques exist to expand on this rather simple approach that allow more information to be derived from each muon using information from the path length of the muon track [4].

\section{Drift Chamber Experiment}

AWE has developed an experimental muon screening system, based on the drift chamber system developed as part of the OPAL experiment, in partnership with Manchester University. Drift chambers were chosen for this particular experimental system as they can be used to cover a large area at a relatively low cost. Several layers of drift chambers were placed above and below the inspection volume of the system to accurately determine the incoming and outgoing trajectory of each cosmic muon. This track information was then used to determine the location of high density materials within the scan volume.

The experimental system was constructed using eight layers of drift chambers, arranged into four detector layers above and below an inspection volume, as shown in Figure 1. These detector layers had a total of four separate parallel drift chambers, each measuring $0.6 \times 1.8 \mathrm{~m}^{2}$ with a central anode wire positioned along the length of the chamber. Contained within each of the drift chambers was a drift gas mixture comprising of $92.5 \%$ argon (Ar), $5 \%$ carbon dioxide $\left(\mathrm{CO}_{2}\right)$ and $2.5 \%$ methane $\left(\mathrm{CH}_{4}\right)$. Each of the eight layers of drift chambers was activated when a muon interacted with this gas releasing a number of electrons that induced a signal in the anode wire. Positioned above the bottom section of the detectors was a trigger panel constructed using a series of scintillating fibres attached to photomultiplier tubes. As a muon interacted with the scintillator it activated the acquisition system which recorded all of the interactions that occurred in each of the drift chambers within a time frame of $40 \mu \mathrm{s}$. The location of the muon interaction was recorded using the time difference between the initial interaction in the scintillating panel and the 


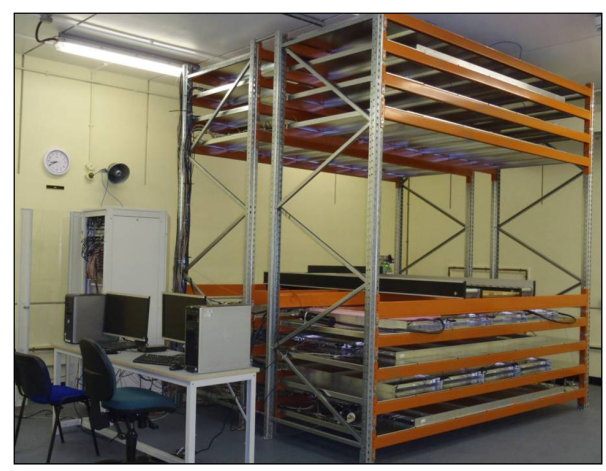

Figure 1: A picture of the drift chamber MST system.

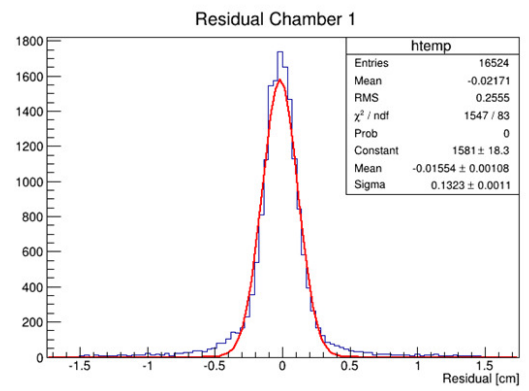

(a) Residual Chamber 1

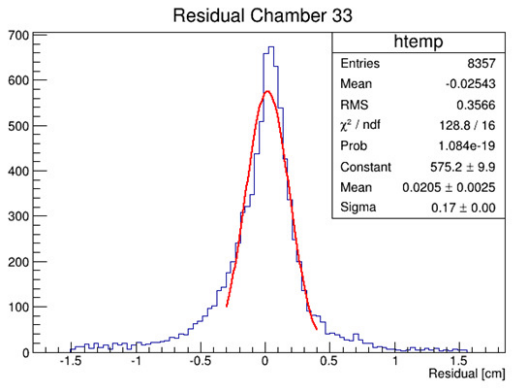

(b) Residual Chamber 33

Figure 2: The residual distribution of muon tracks passing through (a) chamber 1 and (b) chamber 33 positioned at the bottom and top of detector respectively.

recording of the pulse in the drift chamber anode wire. This information was used to reconstruct the trajectories of the muon as it entered and left the inspection volume. The detector assembly was supported by an aluminium frame, which was designed in such a manner that a muon could pass through the drift chambers without interacting with the frame.

At AWE the particle tracking algorithm, used to construct the trajectory of a cosmic muon passing through the detector assembly, determined the muon track from the interaction points in all eight of the detection layers. As each detector plane only recorded the interaction position perpendicular to the wire, sequential layers of drift chambers were arranged in alternating orientations to procedure a three-dimensional track reconstruction. Using this experimental configuration there were four available interaction points to reconstruct the incoming and outgoing muon trajectory. Due to the requirement of a muon being detected in each detector layer the observed detection rate was an order of magnitude lower than the total observed muon flux at sea level.

\section{Experimental Results}

In order to produce an accurate image of the inspection volume it was necessary to reconstruct the incoming and outgoing trajectory of the muon precisely. A detector resolution of 1-2 mm was 


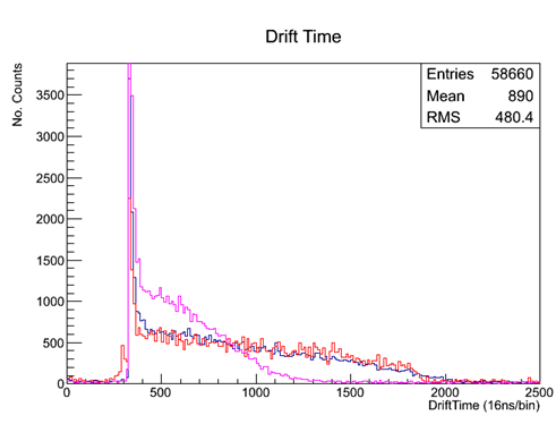

(a) Drift Time Chamber 4

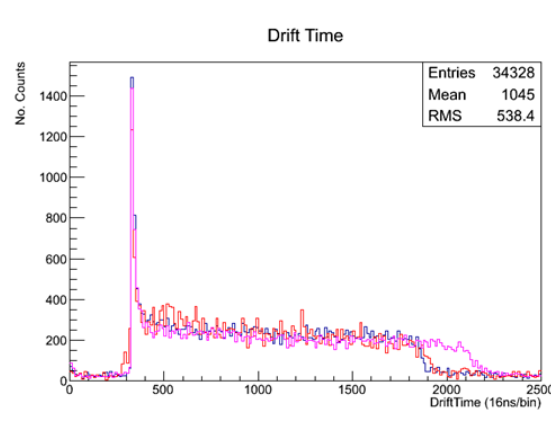

(b) Drift Time Chamber 14

Figure 3: (a) The drift time histogram of chamber 14 before and after correcting the gas leak. (b) The drift time histogram of chamber 4 before and after correcting the gas flow rate issues.

required to achieve the level of precision required. The resolution of the AWE drift chamber system was determined from the track residual information. This residual was determined for each detector using the difference between the recorded and measured point of the muon trajectory, with the point being measured removed from the track fit. The results for two of the drift chambers, positioned near the bottom and top of the drift chamber stack, are shown in Figure 2. These results show that the drift chamber detector system was capable of producing tracks with the necessary position resolution to determine the muon scattering.

During earlier studies of the drift chamber system a number of issues were identified in accurately determining the time taken for the electrons to drift towards the anode wire in each of the individual drift chambers. Two chambers were found to be leaking air into the gas volume, quenching the electron drift and causing a steep drop off in the number of muon interactions recorded at larger distances from the anode wire, an example of this is shown for chamber 14 in Figure 3. After mending the gas leak the drift time histogram reached a plateau after an initial spike of events close to the anode wire, as expected. There was also a flow rate stability issue that affected chamber 4 that was mended by replacing the external gas supply tubing to the particular chamber. A calibration procedure for the detector system was also devised to identify the correct position of each chamber wire within the system using empty target data. This procedure also took into account rotations in the chamber wire and the slight variations in drift velocity between each chamber.

A tungsten cube of side $8.5 \mathrm{~cm}$ with an additional $5 \times 5 \times 7.5 \mathrm{~cm}^{3}$ of tungsten material at either side of the cube, was placed into the inspection volume of the experimental system supported by a common workstation desk, as shown in Figure 4(a). A number of tracks were recorded passing through this inspection using the system. This data was then reconstructed using a PoCA technique to identify the location of the tungsten block. The results of this analysis are shown in Figure 4(b). There is clearly an area of concentrated PoCA points where the tungsten was placed with some areas of higher scattering around the edges of the volume.

\section{Conclusions}

The drift chamber detector system developed by AWE and Manchester University was able to 


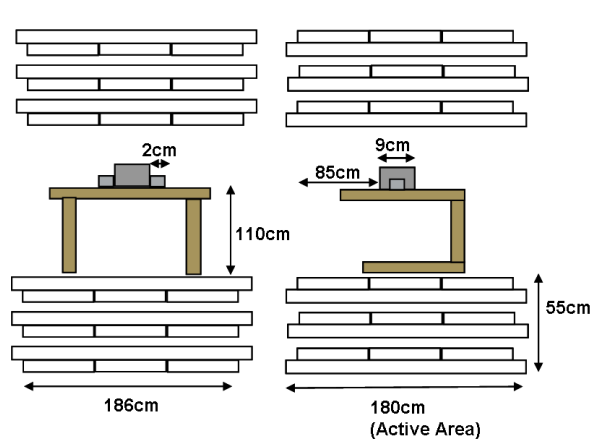

(a) Experiment Setup

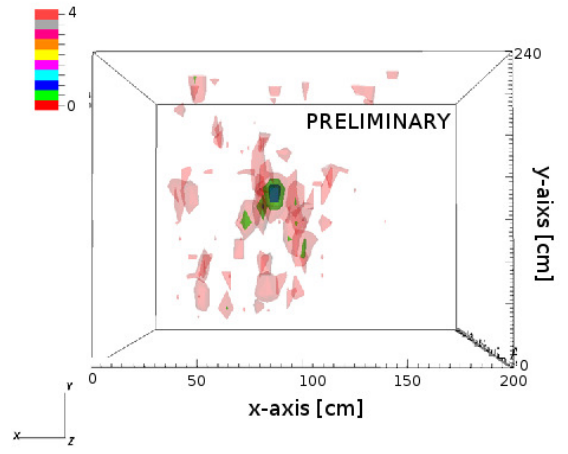

(b) Tungsten image

Figure 4: (a) The experimental setup of tungsten material between the drift chambers. (b) The reconstructed image of the tungsten material.

track cosmic ray muons with sufficient accuracy to identify the presence and location of a block of tungsten situated within the inspection volume of the system. This proof of concept experiment was used to determine the detector attributes that affected the accuracy of the muon track reconstruction within the drift chamber gas volume. This information will be used to enhance future detector design and further develop the track reconstructed and calibration software. These results have also shown that it should be possible to further expand the detector area of this system to cover a larger volume which could be used to scan cargo containers and identify areas of varying density therein.

\section{References}

[1] J. Beringer et al. (Particle Data Group) Review of Particle Physics 86, 010001 (2012)

[2] C.L. Morris et al. Tomographic Imaging with Cosmic Ray Muons Science \& Global Security The Technical Basis for Arms Control, Disarmament, and Nonproliferation Initiatives, 16, 37-53 (2008)

[3] K. Brozdin et al. Cosmic-ray muon tomography and its application to the detection of high-z materials Proceedings of the 46th Annual Meeting, Institute of Nuclear Materials Management (2005)

[4] L.J. Schultz et al. Statistical reconstruction for cosmic ray muon tomography IEEE transactions on imaging processing, vol. 16 , N. 8, (2007) 